\title{
Effect of Early Mobilization on Hip and Lower Extremity Postoperative: A Literature Review
}

\author{
${ }^{1,2}$ Aprisunadi, ${ }^{3}$ Moses Glorino Rumambo Pandin \\ ${ }^{1}$ Doctoral Program Faculty of Nursing, Universitas Airlangga \\ ${ }^{2}$ Lectuter at Faculty of Health Science, Universitas Respati Jakarta \\ ${ }^{3}$ Lecturer at Faculty of Humanities, Universitas Airlangga \\ Email: aprisunadi-2020@fkp.unair.ac.id,moses.glorino@fib.unair.ac.id
}

\begin{abstract}
Background: The incidence of fracture and surgery of the hip and lower extremities is still high. Long postoperative bed rest has the potential to increase the incidence of various complications that may increase the morbidity and mortality rate of patients after hip and lower extremities surgery.

Aim: This literature review aimed to identify the effects of early mobilization in hip and lower extremity postoperative.

Method: Search for articles on several databases such as ProQuest, ScienceDirect, CINAHL, Medline, Wiley Online and Scopus, using the Boolean operator by combining several keywords according to the literature review topic, with inclusion criteria are published in the last 3 years (2019-2021), used a quantitative design, written in English and full text articles. A total of 435 articles were obtained, then screened and reviewed, so that there were 16 (sixteen) eligible articles.

Result: There were 11 (eleven) effects of early mobilization, i.e. reducing length of stay, reducing the risk of deep vein thrombosis (DVT), reducing pulmonary infections (pneumonia), reducing urinary tract infections, reducing the risk of wound infection, improving extremity function and ability to walk, reducing postoperative pain, reducing the risk of pressure sores, reducing postoperative delirium, reducing readmission and lowering hospitalization costs.

Conclusion: This literature review showed that early mobilization is safe and effective in postoperative patients to reduce the risk of complications and adverse events. Nurses and health workers who care for patients could implement early mobilization and motivate patients to be cooperative in undergoing early mobilization.
\end{abstract}

Keywords: Early mobilization, effect, hip, lower extremity, postoperative

\section{BACKGROUND}

The incidence of hip and lower extremity fractures is still high, occur about 100 per 100,000 peoples each year (1). Surgery on hip and lower extremity, especially on the femur and knee are also increasingly common, that the majority of patients undergoing surgery are elderly who are accompanied by various comorbid. Although surgical and anesthetic techniques have improved, morbidity and mortality rate following pelvic and lower extremity surgery are still high (2-4). The high morbidity rate is found on half of patients with hip fractures who are unable to achieve the pre-operative ability to carry out daily activities (5). The one-year mortality rate of hip surgery about $12-33 \%$ and the mortality rate about 8 times in patients 
above 80 years old (1). In hip fracture postoperative, the mortality rate was $37.1 \%$ in men and $26.4 \%$ in women (6).

This high morbidity and mortality rate is associated with some complications that occur due to prolonged bed rest after surgery (1). Complications that may occur such as heart failure, thromboembolism, pneumonia, pressure ulcers, wound healing disorders and delirium $(2,7)$. Early mobilization is considered may reduce postoperative complications due to prolonged bed rest and has some benefits, so that early mobilization widely implemented. Early mobilization is defined as movement of the lower extremities performed within 24 hours of surgery (3).

Early mobilization not only affect short-term outcomes, such as reduced complication and shortened length of stay, but also affect long-term outcome such as increased autonomy and reduced mortality (8). Early mobilization is not without risks. Early mobilization can increase the risk of falls and patient discomfort, so have to carefully implemented (9). Therefore, a literature review is needed to identify the effects of early mobilization in hip and lower extremity postoperative.

\section{METHOD}

\section{Searching Strategy}

This literature review used articles from databases, i.e. ProQuest, Science Direct, CINAHL, Medline, Wiley Online, and Scopus. Search for relevant articles using Boolean operator, such as word 'AND' and 'OR' by combining several key words as follows:

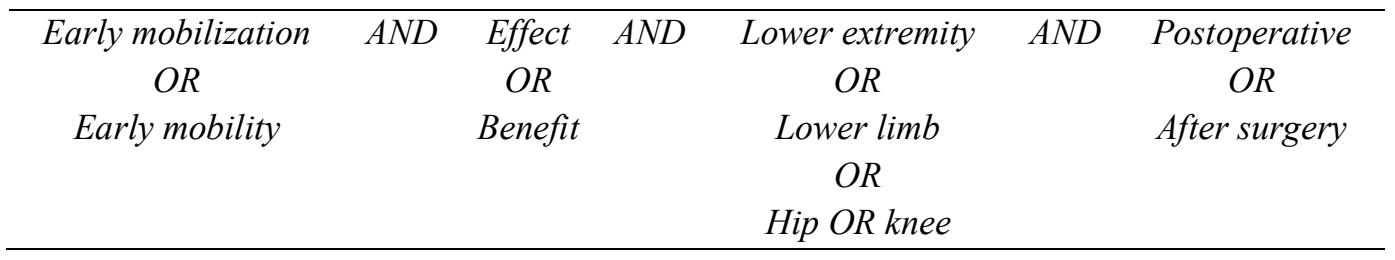

\section{Inclusion Criteria}

The articles used in this literature review are published in the last 3 years (2019-2021), use a quantitative design, have full text and use English. The inclusion criteria applied to the sample or population of articles:

- $\quad$ Adult patient $(\geq 18$ years old $)$

- Underwent early ambulation ( $\leq 24$ hours postoperative)

- Not caused by tumor (malignancy)

\section{RESULT}

The article search obtains 435 articles. 272 articles from ProQuest, 42 articles from ScienceDirect, 29 articles from CINAHL, 57 articles from Medline, 26 articles from Wiley Online and 9 articles from Scopus. The research articles were screened and reviewed based on inclusion criteria and relevance to the topics, 16 (sixteen) articles were obtained. These 
articles showed that there are 11 (eleven) effects of early mobilization, with the following descriptions:

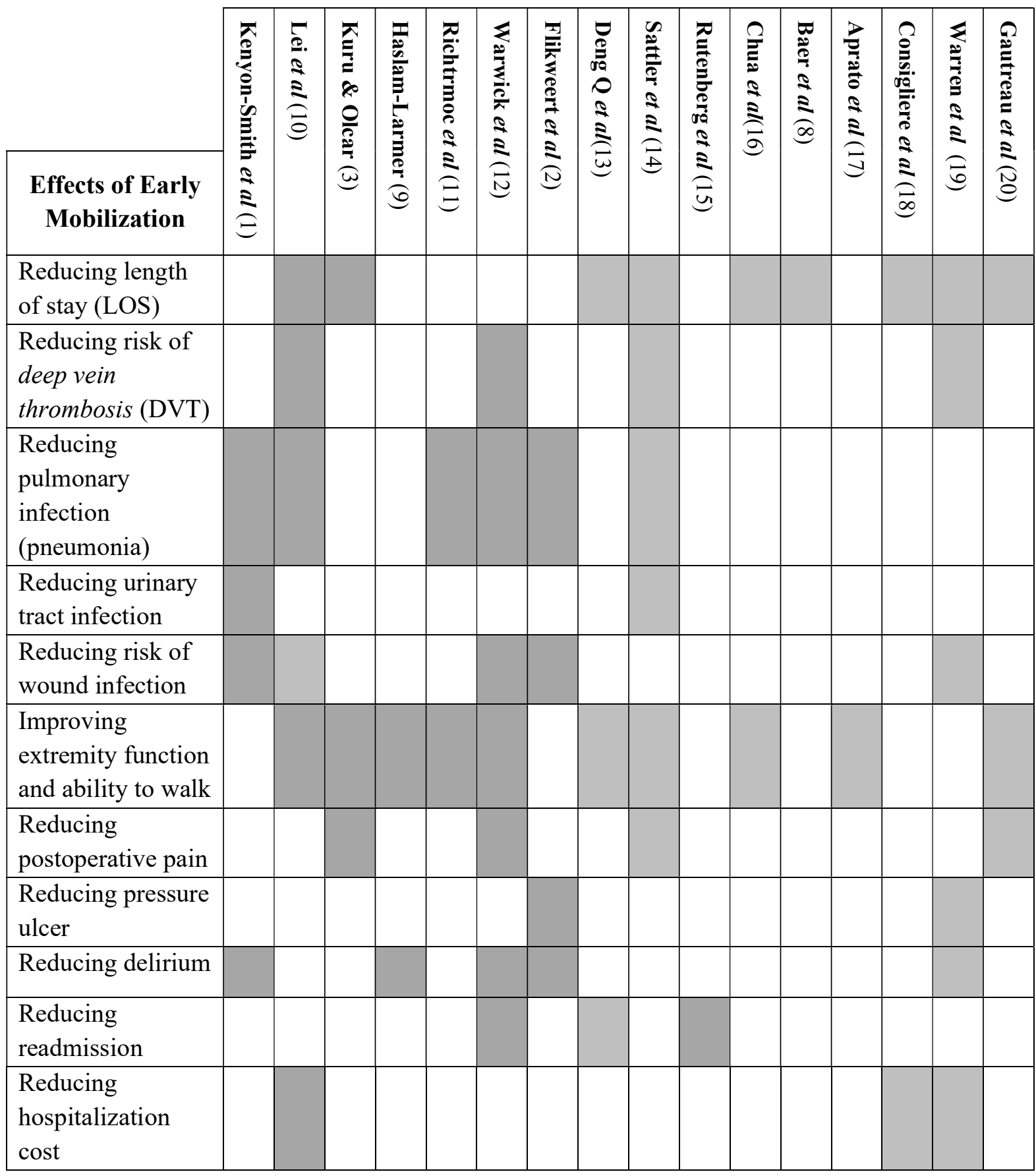

\section{DISCUSSION}

\section{Reducing Length of Stay (LOS)}

The reduction in length of stay is associated with decrease in the incidence of postoperative complications that occur if the patient does not undergo early postoperative mobilization early 
$(3,10,13,14)$. In addition, early mobilization is also able to accelerate the recovery process after patient undergoing hip surgery $(8,9)$. The length of stay on patients with total knee arthroplasty (TKA) that undergo early mobilization are shorter than patients did not undergo early mobilization (10 days versus 12 days) (10). Then, early mobilization of femur postoperative patients also showed an acceleration of the length of stay (18).

The length of stay of knee and hip postoperative patients that undergo early mobilization appeared to be shorter than in patients did not undergo early mobilization (16,20). Delayed mobilization after pelvic fracture surgery can lead to several complications such as pneumonia, urinary tract infections, thromboembolism and delirium that can prolong length of stay (3).

\section{Reducing Risk of Deep Vein Thrombosis (DVT)}

The incidence of DVT is lower in patients undergoing early mobilization compared to patients who did not undergoing early mobilization ( $0,71 \%$ versus $1,41 \%)(10)$. To minimize the risk of DVT, patients should be encouraged to load at least $50 \%$ of their total body weight on the injured extremity after 1 week of surgery (21). Early mobilization is recommended for hip fracture postoperative patients to prevent DVT (19).

\section{Reducing Pulmonary Infection (Pneumonia)}

The incidence of pneumonia is more common in patients who do not undergo early mobilization. Pneumonia only occurred in $0.2 \%$ patients who undergo early mobilization, whereas in patients who do not undergo early mobilization, $0.64 \%$ of patients have pneumonia (10). The incidence of pneumonia can be reduced because early mobilization can effectively improve the strength and function of the respiratory muscles (11).

\section{Reducing Urinary Tract Infection}

Prolong bed rest can lead to unexpected events, one of them is urinary tract infection (3). Early mobilization can prevent urinary retention and thereby reducing the incidence of urinary tract infections (14). In addition, prolonged bed rest can cause negative effects on the urinary tract, one of them is urinary tract infection (1). Urinary tract infection is also recognized as the second biggest reason (subsequent of pneumonia) that causes patient readmission after pelvic surgery (15). In total knee replacement (TKR) patients who undergo early mobilization have a shorter duration of urinary catheter insertion, so that the risk of urinary tract infections is decreased (14).

\section{Reducing Risk of Wound Infection}

The risk of wound infection increases as a result of dehiscence or reopening of the surgical wound. The incidence of wound dehiscence is lower in TKA patients who undergo early mobilization $(0.22 \%)$, compared to TKA patients who do not undergo early mobilization $(0.35 \%)(10)$. 


\section{Improving Extremity Function and Ability to Walk}

Extremity function and ability to walk in patients who undergo early mobilization are found to be better than in patients who do not undergo early mobilization. About $78.3 \%$ patients of postoperative pelvic fracture who are able to walk with or without assistive devices after early mobilization, and only about $21.7 \%$ patients are able to walk who do not undergo early mobilization (3).

\section{Reducing Postoperative Pain}

TKA patients who undergo early mobilization require a low analgatic (morphine) dose compared with TKA patients who do not undergo early mobilization. Total joint arthroplasty (TJA) postoperative patients who undergo early mobilization have better pain control than patients who do not undergo early mobilization (12). Early mobilization can be an intervention for pain management in femur postoperative patients (17). Pain management in hip fractures should be carried out adequately, especially in patients with trochanter fractures (8).

\section{Reducing Pressure Ulcer}

Pressure ulcer is found to occur in $34.2 \%$ or one-third of pelvic surgery patients experience pressure ulcer at follow-up. This pressure ulcer complication is commonly found in elderly patients who have undergone pelvic surgery $(10,22)$. The occurrence of postoperative pressure ulcer of hip fracture will slow down the recovery process and become a predictor of patient mortality (23).

\section{Reducing Delirium}

Delirium is the most common complication (about 61\%) in hospitalized hip fracture patients. Patients not only experience physiological stress due to surgery, but also experience cognitive frailty which causes the patient to experience delirium. Delirium is a risk factor for increased mortality, poor prognosis and a risk of experiencing readmission $(1,2,15)$.

Patients who develop postoperative delirium have a 2.3 times chance of developing other complications. Early mobilization and delirium are interrelated. Early mobilization can reduce the complications of delirium, and delirium can also reduce the chance of the patients undergoing early mobilization (1).

\section{Reducing Readmission}

The 30-day readmission rate is lower in TJA postoperative patients who undergo early mobilization. Readmission occurred in $3.1 \%$ patients who undergo early mobilization and $7.6 \%$ patients who do not undergo early mobilization (12). Early mobilization can prevent complications and improve quality of life, thereby reducing readmission $(2,15)$.

\section{Reducing Cost of Hospitalization}

TKA patients who undergo early mobilization spent lower hospitalization costs compared to TKA patients who do not undergo early mobilization. Early mobilization can shorten the 
length of stay, reduce complications and improve bodily functions, thereby reducing treatment costs (10). Early mobilization in hip fracture postoperative shows a lower incidence of complications. Mobilization that is carried out more than 24 hours is a predictor of an increase in the complication risk (8). The increase in the cost of hospitalization is also largely determined by the lengthening of the patient's length of stay after femoral fracture surgery (25).

\section{CONCLUSION}

The incidence of complications after hip and lower extremities surgery is still quite high, leading to prolonged length of stay, increased morbidity and mortality, increased unplanned readmissions and increased costs of hospitalization. These complications and unwanted events can be prevented by programmed early mobilization. Various research articles showed that early mobilization is safe and effective for postoperative patients. Nurses and health providers involved in caring for patients need to equip themselves with the knowledge and ability to provide early mobilization for postoperative patients safely and effectively. Nurses and health providers also need to motivate patients to be cooperative in undergoing early mobilization so that they can optimize the benefits of early mobilization to prevent complications and improve the recovery process.

\section{REFFERENCES}

1. Kenyon-Smith T, Nguyen E, Oberai T, Jarsma R. Early Mobilization Post-Hip Fracture Surgery. Geriatric Orthopaedic Surgery \& Rehabilitation. 2019;10:215145931982643.

2. Flikweert ER, Wendt KW, Diercks RL, Izaks GJ, Landsheer D, Stevens M, et al. Complications after hip fracture surgery: are they preventable? European Journal of Trauma and Emergency Surgery. 2018;44(4):573-80.

3. Kuru T, Olcar HA. Effects of early mobilization and weight bearing on postoperative walking ability and pain in geriatric patients operated due to hip fracture: A retrospective analysis. Turkish Journal of Medical Sciences. 2020;50(1):117-25.

4. Jennison T, Yarlagadda R. A case series of patients change in mobility following a hip fracture. European Journal of Orthopaedic Surgery and Traumatology [Internet]. 2019;29(1):87-90. Available from: https://doi.org/10.1007/s00590-018-2267-y

5. Svenøy S, Watne LO, Hestnes I, Westberg M, Madsen JE, Frihagen F. Results after introduction of a hip fracture care pathway: comparison with usual care. Acta Orthopaedica. 2020;91(2):139-45.

6. Kannegaard PN, van der Mark S, Eiken P, Abrahamsen B. Excess mortality in men compared with women following a hip fracture. National analysis of comedications, comorbidity and survival. Age and Ageing. 2010;39(2):203-9.

7. Song J, Zhang G, Liang J, Bai C, Dang X, Wang K, et al. Effects of delayed hip replacement on postoperative hip function and quality of life in elderly patients with femoral neck fracture. BMC Musculoskeletal Disorders. 2020;21(1):1-8.

8. Baer M, Neuhaus V, Pape HC, Ciritsis B. Influence of mobilization and weight bearing on in-hospital outcome in geriatric patients with hip fractures. Sicot-J. 2019;5(1):4.

9. Haslam-Larmer L, Donnelly C, Auais M, Woo K, DePaul V. Early mobility after fragility hip fracture: a mixed methods embedded case study. BMC Geriatrics. 2021;21(1):1-15.

10. Lei YT, Xie JW, Huang Q, Huang W, Pei FX. Benefits of early ambulation within $24 \mathrm{~h}$ after total knee arthroplasty: a multicenter retrospective cohort study in China. Military Medical Research. $2021 ; 8(1): 1-8$.

11. Richtrmoc MK, Souza Leite W, Martins Azevedo A, Farias Correia R, de Aquino Coelho Lins R, Alencar Lima W, et al. Effect of Early Mobilization on Respiratory and Limb Muscle Strength and Functionality of Nonintubated Patients in Critical Care: A Feasibility Trial. Critical Care Research and Practice. 2020;2020:1-9. 
12. Warwick H, George A, Howell C, Green C, Seyler TM, Jiranek WA. Immediate Physical Therapy following Total Joint Arthroplasty: Barriers and Impact on Short-Term Outcomes. Advances in Orthopedics. 2019;2019.

13. Deng QF, Gu HY, Peng WY, Zhang Q, Huang ZD, Zhang C, et al. Impact of enhanced recovery after surgery on postoperative recovery after joint arthroplasty: Results from a systematic review and metaanalysis. Postgraduate Medical Journal. 2018;94(1118):678-93.

14. Sattler L, Hing W, Vertullo C. Changes to rehabilitation after total knee replacement. Australian Journal of General Practice. 2020;49(9):587-91.

15. Frenkel Rutenberg T, Rutenberg R, Vitenberg M, Cohen N, Beloosesky Y, Velkes S. Prediction of readmissions in the first post-operative year following hip fracture surgery. European Journal of Trauma and Emergency Surgery [Internet]. 2020;46(5):939-46. Available from: http://dx.doi.org/10.1007/s00068-018-0997-5

16. Chua H, Brady B, Farrugia M, Pavlovic N, Ogul S, Hackett D, et al. Implementing early mobilisation after knee or hip arthroplasty to reduce length of stay: a quality improvement study with embedded qualitative component. BMC Musculoskeletal Disorders. 2020;21(1):1-14.

17. Aprato A, Bechis M, Buzzone M, Bistolfi A, Daghino W, Massè A. No rest for elderly femur fracture patients: early surgery and early ambulation decrease mortality. Journal of Orthopaedics and Traumatology [Internet]. 2020;21(1). Available from: https://doi.org/10.1186/s10195-020-00550-y

18. Consigliere P, Iliopoulos E, Ads T, Trompeter A. Early versus delayed weight bearing after surgical fixation of distal femur fractures: a non-randomized comparative study. European Journal of Orthopaedic Surgery and Traumatology [Internet]. 2019;29(8):1789-94. Available from: https://doi.org/10.1007/s00590-019-02486-4

19. Warren J, Sundaram K, Anis H, McLaughlin J, Patterson B, Higuera CA, et al. The association between weight-bearing status and early complications in hip fractures. European Journal of Orthopaedic Surgery and Traumatology [Internet]. 2019;29(7):1419-27. Available from: https://doi.org/10.1007/s00590-019-02453-z

20. Gautreau S, Haley R, Gould ON, Canales DD, Mann T, Forsythe ME. Predictors of farther mobilization on day of surgery and shorter length of stay after total joint arthroplasty. Canadian Journal of Surgery. 2020;63(6):E509-16.

21. Aufwerber S, Heijne A, Edman G, Grävare Silbernagel K, Ackermann PW. Early mobilization does not reduce the risk of deep venous thrombosis after Achilles tendon rupture: a randomized controlled trial. Knee Surgery, Sports Traumatology, Arthroscopy [Internet]. 2020;28(1):312-9. Available from: https://doi.org/10.1007/s00167-019-05767-x

22. Zaki HAE, Mousa SM, el Said SMS, Mortagy AK. Morbidity and Mortality following Surgery for Hip Fractures in Elderly Patients. Journal of Aging Research. 2019;2019:9-11.

23. Morri M, Ambrosi E, Chiari P, Orlandi Magli A, Gazineo D, D’ Alessandro F, et al. One-year mortality after hip fracture surgery and prognostic factors: a prospective cohort study. Scientific Reports. 2019;9(1):1-8.

24. Agrawal S, Turk R, Burton BN, Ingrande J, Gabriel RA. The association of preoperative delirium with postoperative outcomes following hip surgery in the elderly. Journal of Clinical Anesthesia [Internet]. 2020;60(April 2019):28-33. Available from: https://doi.org/10.1016/j.jclinane.2019.08.015

25. Sermon A, Rochus I, Smeets B, Metsemakers WJ, Misselyn D, Nijs S, et al. The implementation of a clinical pathway enhancing early surgery for geriatric hip fractures: how to maintain a success story? European Journal of Trauma and Emergency Surgery [Internet]. 2019;45(2):199-205. Available from: http://dx.doi.org/10.1007/s00068-018-1034-4 